\title{
Ability of the Nociception Level, a Multiparameter Composite of Autonomic Signals, to Detect Noxious Stimuli during Propofol-Remifentanil Anesthesia
}

\author{
Chris H. Martini, M.D., Martijn Boon, M.D., Suzanne J. L. Broens, M.D., Evelien F. Hekkelman, M.D., \\ Lisanne A. Oudhoff, M.D., Anna Willemijn Buddeke, M.D., Albert Dahan, M.D., Ph.D.
}

\begin{abstract}
Background: A novel multidimensional index of nociception, the nociception level (NoL) index, derived from the nonlinear composite of heart rate (HR), HR variability, amplitude of the photoplethysmogram, skin conductance, fluctuations in skin conductance, and their time derivatives, was used to assess nociception during anesthesia.

Methods: Seventy-two American Society of Anesthesiologists I to III patients received propofol (target bispectral index, 45) and one of six remifentanil target concentrations in the range of 0 to $5 \mathrm{ng} / \mathrm{ml}$. The NoL, mean arterial pressure (MAP; derived from a beat-to-beat finger cuff measurement), and HR were measured during nonnoxious, moderate noxious (skin incision), and intense noxious (intubation) stimulation. Values are represented as average (95\% CI).

Results: NoL, HR, and MAP did not change during nonnoxious events. Incision increased HR by $1.3 / \mathrm{min}(-0.46$ to $3.1 / \mathrm{min}$; not significant), MAP by $7.9 \mathrm{mmHg}$ ( -1.9 to $13.0 \mathrm{mmHg}$; not significant), and NoL by $8.0(0.4$ to $16.0 ; P<0.001)$. Intubation increased HR by $7.0 / \mathrm{min}(1.4$ to $12.0 / \mathrm{min} ; P<0.001)$, MAP by $13.0 \mathrm{mmHg}(3.1$ to $20 \mathrm{mmHg} ; P<0.001)$, and NoL by 18.0 (7.8 to $29.0 ; P<0.001)$. The $\Delta \mathrm{NoL}$ area under the curve $(0.95)$ of the receiver operating characteristic curve was greater compared with $\triangle \mathrm{HR}(0.84, P<0.001)$ and $\triangle \mathrm{MAP}(0.78, P<0.001)$. Under nonnociceptive conditions, remifentanil had no effect on NoL, in contrast to HR and MAP that showed a dose-dependent decrease.

Conclusions: The NoL is a reliable measure of moderate and intense noxious stimulation and outperforms HR and MAP in differentiating noxious from nonnoxious stimuli. In contrast to HR and MAP, the NoL was not affected by hemodynamic effects of remifentanil. (Anesthesiology 2015; 123:00-00)
\end{abstract}

\begin{abstract}
A CCURATE measurement of nociception during anesthesia remains a challenging task. Nociception, which is defined as the neural process of encoding and processing noxious stimuli (noxious stimuli are actually or potentially tissue damaging events), ${ }^{1}$ will elicit behavioral, autonomic, and hormonal responses in conscious and unconscious individuals. Detection of behavioral responses during anesthesia is often impossible because of the use of muscle relaxants. Hence, we rely mostly on the autonomic responses to assess the nociception level (NoL) of the patient. Most anesthesia healthcare providers, if not all, use changes in heart rate (HR) and blood pressure as markers of the occurrence of acute nociceptive events. Although these variables may suffice when intense nociceptive stimuli occur, mild and moderate stimuli are often not detected or detected too late. ${ }^{2}$

In recent years, various indices of nociception have been developed with varying success in actually detecting nociceptive events. These indices derive a numerical value from single variables (such as heart rate variability [HRV], skin conductance, skin vasomotor reflex, the electroencephalogram,
\end{abstract}

\section{What We Already Know about This Topic}

- We lack well-validated methods for following the level of nociception during anesthesia

\section{What This Article Tells Us That Is New}

- A multidimensional index of nociception level was developed using patients under propofol anesthesia receiving remifentanil infusions at a range of rates

- The nociception level index was found to be a reliable measure of noxious stimulation and outperformed simpler measures

pupil diameter) or a combination of signals. ${ }^{3-11}$ In the current study, we apply a new index of nociception, the NoL index. ${ }^{2}$ The NoL is a multiparameter nonlinear combination of HR, HRV, amplitude of the finger photoplethysmogram (AP), skin conductance level, fluctuations in skin conductance, and their time derivatives, derived from random forest regression. Random forest is an algorithmic modeling approach that enables combining multiple parameters of different origin and discovering their complex nonlinear

This article is featured in "This Month in Anesthesiology," page 1A. Supplemental Digital Content is available for this article. Direct URL citations appear in the printed text and are available in both the HTML and PDF versions of this article. Links to the digital files are provided in the HTML text of this article on the Journal's Web site (www.anesthesiology.org). Full protocol available at: a.dahan@lumc.nl. Raw data available at: a.dahan@lumc.nl.

Submitted for publication November 24, 2014. Accepted for publication May 16, 2015. From the Department of Anesthesiology, Leiden University Medical Center, Leiden, The Netherlands.

Copyright $\odot$ 2015, the American Society of Anesthesiologists, Inc. Wolters Kluwer Health, Inc. All Rights Reserved. Anesthesiology 2015; 123:00-00 
interactions. ${ }^{12,13}$ We measured the NoL, HR, and arterial blood pressure during induction of general propofol-remifentanil anesthesia, intubation, and incision. Our aims were to validate the NoL as measured in real time by assessing its ability to detect moderate and intense nociceptive stimuli under different target remifentanil blood concentrations. The NoL was compared with the more commonly used indices of nociception, mean arterial pressure (MAP), and HR.

\section{Materials and Methods}

The protocol was performed after obtaining approval from the local Human Ethics Committee (Commissie Medische Ethiek, Leiden University Medical Center, Leiden, The Netherlands) and was registered at www.clinicaltrials.gov under number NCT01912118. All patients gave oral and written informed consent before enrolment into the study. The study was performed from July 2013 to June 2014.

\section{Patients}

American Society of Anesthesiology class I, II, or III patients (age, 18 to $80 \mathrm{yr}$ ) of either sex, scheduled for elective surgery under general anesthesia, were recruited to participate in the study. Exclusion criteria included inability to give informed consent, pregnancy or lactation, body mass index more than $35 \mathrm{~kg} / \mathrm{m}^{2}$, perceived difficult intubation, planned rapid sequence intubation, and use of $\beta$-adrenergic receptor antagonists. Preoperative preparation was according to local protocol.

\section{Study Design}

In this prospective randomized study, patients received total intravenous anesthesia with propofol and remifentanil. Seventy-two patients were randomly assigned to one of six possible remifentanil target concentrations: 0 (propofol only, $\mathrm{n}=12), 1,(\mathrm{n}=12), 2(\mathrm{n}=12), 3(\mathrm{n}=12), 4(\mathrm{n}=12)$, and $5(\mathrm{n}=12) \mathrm{ng} / \mathrm{ml}$, using a custom-built remifentanil target controlled infusion pump (Remifusor, University of Glasgow, United Kingdom) programmed with the remifentanil pharmacokinetic dataset published by Minto et al. ${ }^{14}$ Similarly, propofol was infused using a target-controlled infusion system (Orchestra Base Primea, Fresenius Kabi, The Netherlands) programmed with the propofol pharmacokinetic dataset published by Marsh et al. ${ }^{15}$ The target was adapted such that before intubation or skin incision the bispectral index (BIS) of the electroencephalogram (BIS ${ }^{\circledR}$ VISTA, Covidien, Ireland) was maintained at $45 \pm 5$ for at least 10 to $15 \mathrm{~min}$. If needed, a muscle relaxant (rocuronium, $0.5 \mathrm{mg} / \mathrm{kg}$ ) could be given before intubation.

In the protocol, there were two additional study groups ( $\mathrm{n}=12$, BIS, 70 ; remifentanil, $3 \mathrm{ng} / \mathrm{ml}$; and $\mathrm{n}=12$, BIS, 30; remifentanil, $3 \mathrm{ng} / \mathrm{ml}$ ). After enrolment of four subjects in this subprotocol, further inclusion of subjects was stopped because of safety concerns (e.g., possibility of awareness, hemodynamic instability).

\section{Data Collection}

A finger probe containing sensors for measurement of the photoplethysmogram, the Galvanic skin response, skin temperature, and three-axis accelerometer was placed on the index finger of the right hand (Medasense Biometrics, Israel). ${ }^{2,16}$ The signals from the probe were sampled at 50 $\mathrm{Hz}$ and recorded on a laptop computer using the PMD-10X system and software (Medasense Biometrics). All data were processed offline using MATLAB R2011b software (The Mathworks Inc., USA). The following variables were calculated from the finger probe as specified by Ben-Israel et al. ${ }^{2}$ : HR, HRV, AP, skin conductance level, and fluctuations in skin conductance. To measure the noninvasive beat-to-beat blood pressure, an appropriately sized finger cuff was applied to the mid-phalanx of the left index finger, which was connected to a Nexfin monitor (Edwards Lifesciences, USA). Refer the study by Martina et al. ${ }^{17}$ for an elaborate explanation of the Nexfin system and calculation of blood pressure. The beat-to-beat finger arterial blood pressure was stored on disc for offline analysis. The PMD-10X and the Nexfin systems were time aligned before each study. Data were collected from induction of anesthesia until 3 to $5 \mathrm{~min}$ after incision. Specific events occurring during the study (start of induction, patient movement, intubation, and incision) were logged in the PMD-10X software program enabling a direct link between stimulus and measurements.

\section{Description of the Nociception Level Index}

The NoL is based on a nonlinear combination of nociception-related physiologic parameters: HR, HRV (at the 0.15 to $0.4 \mathrm{~Hz}$ band power), amplitude of the photoplethysmograph wave, skin conductance level, number of skin conductance fluctuations, and their time derivatives. ${ }^{2}$ The $\mathrm{NoL}$ index was developed to correlate with a reference clinical score of nociception based on the estimated opioid concentration and stimulus strength (i.e., the combined index of stimulus and analgesia [CISA]). A composite parameter was derived from random forest analysis, ${ }^{12,13}$ a nonlinear regression method, in which the physiologic signals with their derivatives were used as predictor variables and the CISA was used as the observed variable. The estimated multiparameter composite derived from the regression analyses was scaled from 0 to 100 to produce the NoL. The NoL index has been shown to provide a better indication of nociception than each of its component physiologic signals and to their linear combination. ${ }^{2}$ In the current study, the NoL index was calculated from the extracted signals by the PMD-10X software/hardware system using the algorithm derived from the learning study by Ben-Israel et al. ${ }^{2}$ The NoL device has not received Food and Drug Administration clearance as yet and is still under investigation.

\section{Data Analysis}

Because this study was the first assessment of the NoL in a clinical perioperative setting using total propofol/ 
remifentanil anesthesia, we were uninformed regarding the possible effect sizes of the three stimuli on the NoL. We somewhat arbitrarily set the sample size at 12 subjects per remifentanil treatment level or 72 patients in total, a number very similar to several previous studies linking physiologic signals to nociception. ${ }^{9,11}$

Statistical and data analyses were performed using MATLAB R2011b software (The Mathworks Inc.). Three distinct stimuli were defined in each patient: a nonnoxious event, incision, and intubation, which were regarded as nonnoxious, moderate noxious, and severe noxious stimuli, respectively. ${ }^{2,18,19}$ We graded the nociceptive intensity, nonnoxious event $>$ incision $>$ intubation, based on the previous studies that showed that the opioid concentration needed to suppress the autonomic response to intubation exceeds the concentration required to suppress the response to skin incision by a factor of $2 .{ }^{18,19}$ A nonnoxious event was defined as a 1 -min interval within a 5 -min window of absence of noxious stimulation; intubation was defined as the time interval around the insertion of the oropharyngeal tube into the trachea and included the preceding laryngoscopy; incision was defined as the time interval around the surgical skin incision. For each stimulus, two parameter values were defined, one before stimulation (before) and one after stimulation (after), which were the average of data before and after the stimulus along a certain time interval. These time intervals were the first (before) and last (after) $30 \mathrm{~s}$ of the 1-min nonnoxious interval for the nonnoxious stimulus and the 30 to 60 s before the stimulus (before) and the 10 to $180 \mathrm{~s}$ after the stimulus (after) for incision and intubation. Analysis was done on both the absolute MAP, HR, and NoL values and the difference between after and before values (i.e., $\Delta)$. In case of use of vasoactive (e.g., ephedrine and phenylephrine) and vagolytic (e.g., atropine) drugs in these time windows, the data were discarded.

\section{Statistical Analysis}

The following statistical tests were performed to compare the performance of NoL, $\triangle$ NoL, MAP, $\triangle \mathrm{MAP}, \mathrm{HR}$, and $\Delta \mathrm{HR}$ :

1. Right-tailed paired $t$ test to assess whether the average reaction $(\Delta)$ of the three variables to stimulation are significantly greater than 0 . Two-tailed unpaired $t$ test to assess whether the population values of the variables after stimulation were significantly different from the values obtained before stimulation. In addition, the effect of stimulation on BIS was tested using paired and unpaired $t$ tests.

2. Receiver operating characteristic (ROC) curves were constructed to assess the ability of the individual variables (absolute values and $\Delta$ ) to discriminate between noxious and nonnoxious events. CIs of the area under the ROC curves (AUCs) were calculated using the method suggested by Hanley and McNeil, ${ }^{20}$ which corrects for the use of correlated data.
3. Repeated measures ANOVA to test the ability of each of the variables to grade noxious stimuli, i.e., to assess whether the variable values increased with an increasing stimulus strength: nonnoxious stimulus $<$ moderate noxious stimulus (incision) $<$ intense noxious stimulus (intubation). In case of a significant main and interaction terms, a Scheffe post hoc multiple comparison test was applied to test between pairs nonnoxious stimulus versus intubation, nonnoxious stimulus versus incision, and incision versus intubation.

4. For nonnoxious stimuli and intubation, the Spearman correlation coefficient was calculated to quantify the relation between HR, MAP, NoL, and the remifentanil target concentration. This was done separately for time intervals before and after stimulation. A quadratic polynomial was fitted by least square analysis to the data.

\section{Results}

Seventy-two patients participated in the study according to protocol (data from the subprotocol are not considered here). The characteristics of the 72 participating patients are given in table 1 . The complete dataset of one subject was lost because of technical problems. The data from 71 patients were used in the analysis. All patients completed the study without side effects. In about half of the patients, a muscle relaxant was administered before intubation. Before noxious stimulation (intubation/skin incision), BIS values were on average of $45.0 \pm 9.0$ (mean \pm SD), $45.6 \pm 9.9,47.2 \pm 9.1$, $42.6 \pm 7.4,44.7 \pm 8.0$, and $47.0 \pm 9.8$ in the $0,1,2,3,4$, and $5 \mathrm{ng} / \mathrm{ml}$ remifentanil groups, respectively (repeated measures ANOVA: $P>0.05$; grand mean, 45.5 \pm 8.8$)$. Because of technical (e.g., monitor failure), logistic (e.g., change of surgical plan), or other reasons (e.g., hypertension/hypotension or bradycardia in response to low/high remifentanil requiring the use of vasoactive/vagolytic drugs; lack of annotations) that caused an inadvertent breach of protocol, the datasets missed one of the noxious/ nonnoxious stimuli in 20 to $25 \%$ of cases. The numbers of excluded events are given in table 2.

\section{Response to Noxious Events}

The effect of nonnoxious stimuli, incision, and intubation on BIS, HR, MAP, and NoL are given in figure 1. Nonnoxious stimuli had no effect on any of the variables when comparing before with after time intervals (mean difference [95\% CI]): $\triangle \mathrm{BIS},-0.1(-0.9$ to 0.7$) ; \Delta \mathrm{HR},-0.13 / \mathrm{min}(-0.7$ to $0.3 / \mathrm{min})$; $\triangle \mathrm{MAP},-0.45 \mathrm{mmHg}(-1.9$ to $2.1 \mathrm{mmHg})$; and $\Delta \mathrm{NoL}$ -1.1 (-3.6 to 2.0). Intubation caused an increase in HR, MAP, and NoL but not BIS: $\triangle B I S, 1.7$ (-3.9 to 6.3; not significant [ns]); $\Delta \mathrm{HR}, 7.0 / \mathrm{min} \quad(1.4$ to $12.0 / \mathrm{min}$; paired $t$ test, $P<0.001$; unpaired $t$ test, $P<0.001$ ); $\triangle$ MAP, 13.0 (3.1 to 20; paired $t$ test, $P<0.001$; unpaired 
Table 1. Patient Characteristics for the Six Study Groups and Performed Surgical Procedures

\begin{tabular}{|c|c|c|c|c|c|c|c|}
\hline & REMI 0 & REMI 1 & REMI 2 & REMI 3 & REMI 4 & REMI 5 & All \\
\hline $\mathrm{N}$ & 12 & 12 & 12 & 12 & 12 & 12 & 72 \\
\hline $\mathrm{M} / \mathrm{F}$ & $6 / 6$ & $3 / 9$ & $9 / 3$ & $4 / 8$ & $3 / 9$ & $8 / 4$ & $33 / 39$ \\
\hline Age, yr (range) & $43(20-75)$ & $57(24-74)$ & 56 (37-69) & $55(21-74)$ & $54(26-76)$ & $54(31-73)$ & $54(20-76)$ \\
\hline Height (cm) & $176 \pm 1$ & $169 \pm 6$ & $181 \pm 9$ & $171 \pm 9$ & $170 \pm 9$ & $176 \pm 13$ & $174 \pm 10$ \\
\hline Weight (kg) & $81 \pm 16$ & $67 \pm 12$ & $84 \pm 15$ & $70 \pm 11$ & $69 \pm 12$ & $79 \pm 16$ & $75 \pm 15$ \\
\hline $\mathrm{BMI}$ & $26 \pm 5$ & $24 \pm 4$ & $26 \pm 4$ & $24 \pm 3$ & $24 \pm 3$ & $26 \pm 4$ & $25 \pm 4$ \\
\hline Heart rate (beats $/ \mathrm{min})^{\star}$ & $79 \pm 10$ & $74 \pm 15$ & $72 \pm 15$ & $73 \pm 10$ & $70 \pm 13$ & $74 \pm 18$ & $73 \pm 14$ \\
\hline $\operatorname{MAP}(\mathrm{mmHg})^{\star}$ & $90 \pm 12$ & $99 \pm 14$ & $96 \pm 14$ & $101 \pm 19$ & $96 \pm 18$ & $100 \pm 10$ & $97 \pm 15$ \\
\hline ASA I (n) & 7 & 5 & 5 & 5 & 9 & 8 & 39 \\
\hline ASA II (n) & 5 & 6 & 7 & 7 & 3 & 4 & 32 \\
\hline ASA III (n) & 0 & 1 & 0 & 0 & 0 & 0 & 1 \\
\hline General surgery (n) & 4 & 4 & 5 & 5 & 4 & 8 & 30 \\
\hline Gynecology (n) & 4 & 4 & 2 & 1 & 1 & 4 & 16 \\
\hline Urology (n) & 3 & 2 & 3 & 2 & 1 & & 11 \\
\hline Orthopedics (n) & 1 & & 1 & & 2 & & 4 \\
\hline ENT (n) & & 1 & & 1 & 2 & & 4 \\
\hline Neurosurgery (n) & & 1 & & & & & 1 \\
\hline Oral surgery (n) & & & 1 & & & & 1 \\
\hline Plastic surgery (n) & & & & 3 & 2 & & 5 \\
\hline
\end{tabular}

All values are represented as mean $\pm \mathrm{SD}$ or numbers $(\mathrm{n})$, except age that is represented as median (range).

* Values obtained at patient screening in the preoperative clinic.

$\mathrm{ASA}=$ American Society of Anesthesiologists; $\mathrm{BMI}=$ body mass index; ENT = ear, nose, and throat surgery; $\mathrm{F}=$ female; $\mathrm{M}=$ male; $\mathrm{MAP}=$ mean arterial pressure; $\mathrm{REMI}=$ remifentanil target concentration.

Table 2. Reason for Missing or Discarded Data

\begin{tabular}{lcc}
\hline Device & $\begin{array}{c}\text { Number of Subjects } \\
\text { Included in the } \\
\text { Analysis }\end{array}$ & $\begin{array}{c}\text { Number of Subjects } \\
\text { with Missing } \\
\text { Data (A/B/C) }\end{array}$ \\
\hline $\begin{array}{l}\text { Nonnoxious stimulus } \\
\text { PMD-10X }\end{array}$ & 63 & \\
Nexfin device & 56 & $0 / 0 / 9$ \\
BIS ${ }^{\circledR}$ monitor & 56 & $7 / 0 / 9$ \\
Incision & & $7 / 0 / 9$ \\
PMD-10X & 58 & \\
Nexfin device & 53 & $1 / 5 / 8$ \\
BIS ${ }^{\circledR}$ monitor & 56 & $3 / 5 / 8$ \\
Intubation & & $3 / 5 / 8$ \\
PMD-10X & 67 & \\
Nexfin device & 65 & $0 / 2 / 3$ \\
BIS ${ }^{\circledR}$ monitor & 66 & $2 / 2 / 3$ \\
\hline
\end{tabular}

$\mathrm{A}=$ technical problems; $\mathrm{B}=$ clinical issues (hypotension/hypertension/ bradycardia); BIS = bispectral index of the electroencephalogram; C = lack of annotation.

$t$ test, $P<0.001)$; and $\Delta$ NoL, 18.0 (7.8 to 29.0; paired $t$ test, $P<0.001$; unpaired $t$ test, $P<0.001)$. Incision had no effect on BIS and HR but caused increases in MAP and NoL, although, in contrast to MAP, the effects on NoL were significant in both paired and unpaired $t$ tests: $\Delta \mathrm{BIS}$, $0.92(-1.2$ to $3.3 ; \mathrm{ns}) ; \Delta \mathrm{HR}, 1.3 / \mathrm{min}(-0.46$ to $3.1 / \mathrm{min}$; ns); $\triangle$ MAP, $7.9 \mathrm{mmHg}(-1.9$ to $13.0 \mathrm{mmHg}$; paired $t$ test, $P<0.001$; unpaired $t$ test, ns); and $\Delta$ NoL, 8.0 (0.4 to 16.0 ; paired $t$ test, $P<0.001$; unpaired $t$ test, $P<0.001)$.

Comparing the three different stimuli (i.e., assuming nonnoxious event $\neq$ incision $\neq$ intubation), a significant main and interaction effect was observed for $\mathrm{HR}, \mathrm{MAP}$, and $\mathrm{NoL}$ after (but not before) stimulation and $\Delta \mathrm{s}$ : $\operatorname{HR~F}(2,96)=9.4$, $P<0.001 ; \Delta$ HR F(2,96) $=27, P<0.0001) ;$ MAP $\mathrm{F}(2,80)=28, P<0.001 ; \Delta \mathrm{MAP} \mathrm{F}(2,80)=19, P<0.0001$; $\operatorname{NoL} \mathrm{F}(2,96)=23, P<0.0001 ; \Delta \mathrm{NoL} F(2,96)=46$, $P<0.0001$. Post hoc analysis showed that only NoL (after stimulation) and $\Delta$ NoL graded the level of noxious intensity with nonnoxious $\mathrm{NoL}<$ incision $\mathrm{NoL}<$ intubation NoL. HR after stimulation and $\triangle \mathrm{HR}$ could not differentiate between nonnoxious stimuli and incision $(P=0.24)$. $\triangle \mathrm{MAP}$ could not discriminate between incision and intubation $(P=0.07)$. See also Supplemental Digital Content 1, tables 1 and 2, http://links.lww.com/ALN/B170.

The ROC curves, calculated $(\mathrm{n}=71)$ for HR, MAP, and NoL (all after stimulation), and $\triangle \mathrm{HR}, \triangle \mathrm{MAP}$, and $\Delta$ NoL, are shown in figures 2 and 3. ROC areas under the curve sensitivity values at a specificity of $75 \%$ are given in table 3. $\Delta \mathrm{NoL}$ outperformed all other variables in ability to discriminate between noxious (intubation or incision) and nonnoxious events with an AUC of 0.95 (95\% CI, 0.91 to 0.99$)$. The $\triangle$ NoL AUC was significantly larger compared with all other variables $(P=0.0003$ vs. $\triangle \mathrm{HR} ; P<0.0001$ vs. $\triangle \mathrm{MAP} ; P<0.0001$ vs. HR; $P=0.00004$ vs. MAP). Moreover, NoL after stimulation outperformed MAP and HR in classifying noxious stimuli (AUC, 0.82; 95\% CI, 0.75 to 0.89 , $P=0.001$ vs. HR; $P=0.035$ vs. MAP). The NoL outperformed $\mathrm{HR}$ and MAP and $\triangle \mathrm{NoL}$ outperformed $\triangle \mathrm{HR}$ and $\triangle \mathrm{MAP}$ in terms of sensitivity, specificity, and positive and negative predictive values for the detection of noxious 

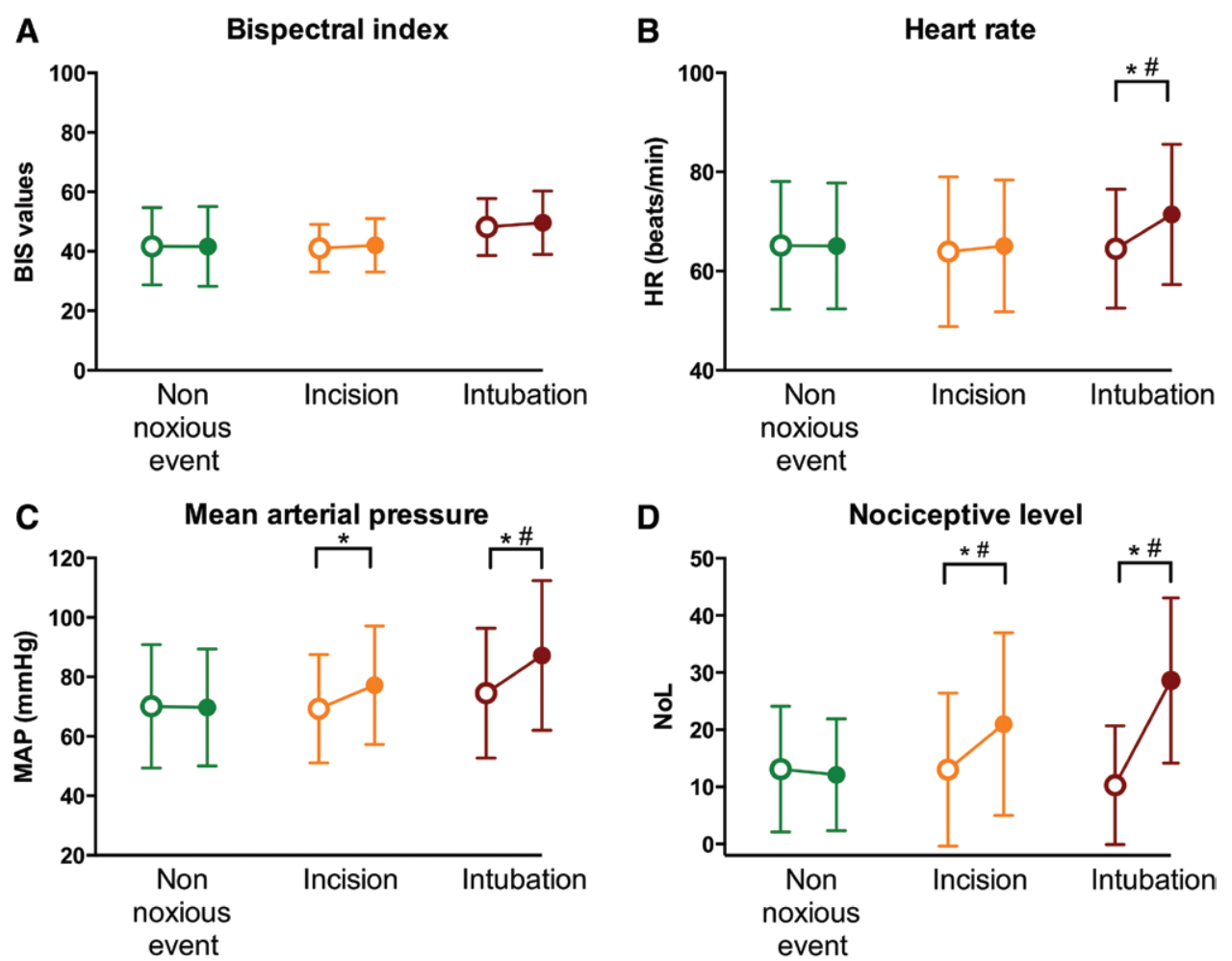

\section{Before stimulation After stimulation}

Fig. 1. Bispectral index $(\mathrm{BIS})(A)$, heart rate $(\mathrm{HR})(B)$, mean arterial pressure (MAP) $(C)$, and nociceptive level $($ NoL) $(D)$ before and after noxious stimulation for nonnociceptive conditions, incision, and intubation. *Paired $t$ test, $P<0.001$; \#unpaired $t$ test, $P<0.001$. Open symbol $=$ before stimulation; closed symbol $=$ after stimulation.

stimuli (table 3 ). For NoL a cutoff value between noxious and nonnoxious stimuli of 16 yielded a specificity and sensitivity of 80 and $73 \%$.

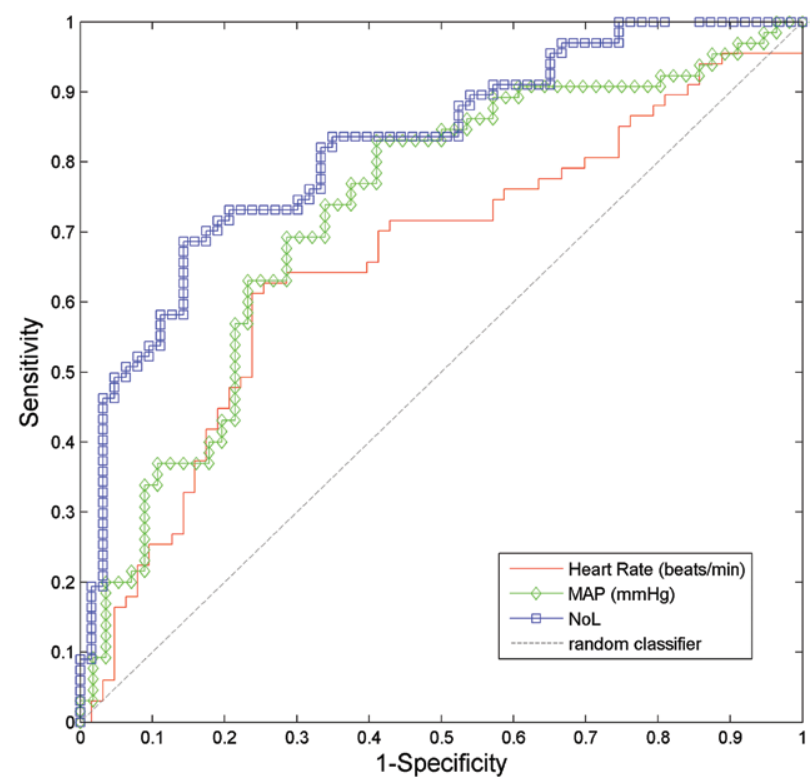

Fig. 2. Discrimination between nociceptive (incision and intubation) and nonnociceptive stimuli: receiver operating curves of heart rate, mean arterial pressure (MAP), and the composite parameter, the nociception level (NoL).

\section{Response to Intubation under Different Remifentanil Target Concentrations}

The effects of increasing concentrations of remifentanil on HR ( $\mathrm{n}=57)$, MAP $(\mathrm{n}=50)$, and NoL $(\mathrm{n}=57)$ before and after noxious stimulation are shown in figures 4 to 6 . The NoL before and after nonnoxious stimulation showed no significant correlation with the remifentanil concentration $\left(r_{S}=-0.047\right.$ and 0.024, P> 0.05; fig. 4, A and B). The before and after intubation NoL values showed a significant Spearman correlation with $r_{S}=-0.3, P<0.05$ (before, fig. 4C) and $\mathrm{r}_{\mathrm{S}}=-0.51, P<0.001$; after, fig. 4D). The analysis indicates that with increasing remifentanil concentrations, the NoL response to intubation decreases significantly with the smallest response observed at a remifentanil target concentration of $5 \mathrm{ng} / \mathrm{ml}$ (fig. 4D). HR before and after nonnoxious stimulation and intubation decreased significantly with increasing remifentanil concentrations $(P<0.01$; fig. 5, A-D). A similar observation was made for MAP before and after nonnoxious stimulation and intubation $(P<0.05$; fig. 6, A-D).

\section{Discussion}

In this validation study, the NoL, a novel multidimensional parameter, was used to detect nociceptive events during propofol-remifentanil anesthesia. The variables that make up the NoL cover both sympathetic and parasympathetic 


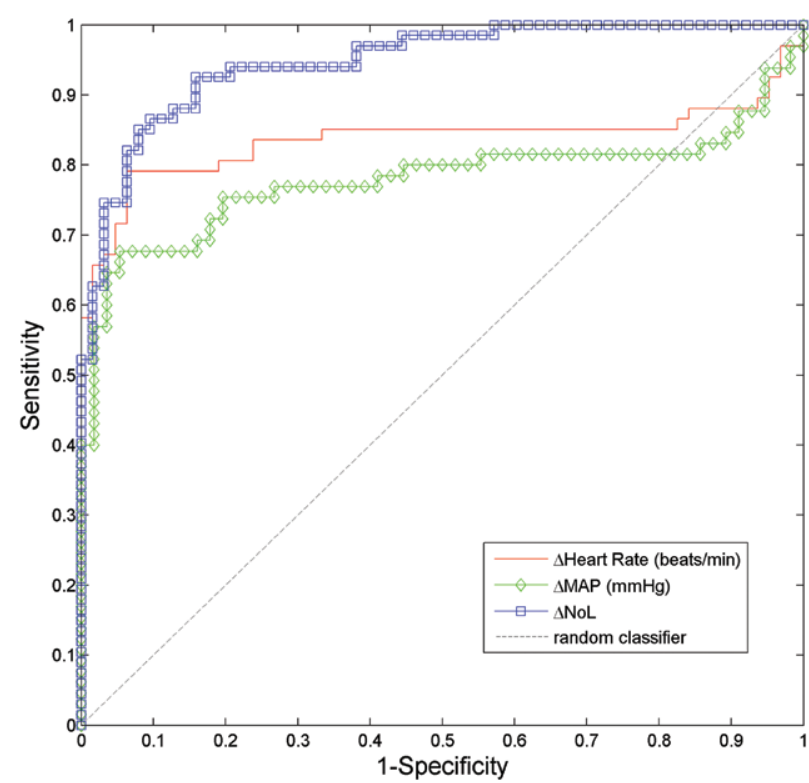

Fig. 3. Receiver operating curves of the hemodynamic $\Delta$ signals and the $\Delta$ nociceptive level $(\Delta \mathrm{NoL}) . \mathrm{MAP}=$ mean arterial pressure.

activities of the autonomous nervous system. ${ }^{21}$ The main observations from our study are that (1) the NoL is able to detect intense noxious stimulation (intubation) and moderate nociceptive stimuli (incision). In this respect, the NoL outperformed the standard variables (HR and MAP) that were sensitive to intubation but to a lesser extent to incision. Furthermore, as based on the AUC of the ROC, the NoL was best in differentiating noxious from nonnoxious stimuli; and (2) the NoL was significantly correlated with the target remifentanil concentration after noxious stimulation. Similar observations were made for HR and MAP; however, in contrast to HR and MAP, the NoL was not affected by nonnoxious events.

Various previous studies have relied on single signals to assess nociception during surgery. ${ }^{3-11,22}$ For example, indices from the high-frequency component of HRV were used to evaluate surgical nociception and analgesia during anesthesia and to predict postoperative pain based on the measurements before extubation. ${ }^{3,22}$ Another example is the measurement of changes in skin conductance, which is based on the sympathetically induced secretion of sweat, which increases skin conductance. ${ }^{4}$ This response (i.e., nociceptionrelated sweating) is unrelated to hemodynamic changes. All single indices aimed at detecting nociception share that they are surrogate markers of the autonomic nervous system and show a large within- and between-subject variability. ${ }^{2,23}$ Another approach in detecting nociception during surgery is the use of multiparameter indices. ${ }^{8-10}$ Several studies show that a multiparameter approach yields greater sensitivity and specificity in discriminating between noxious and nonnoxious stimuli than the single-signal approach. ${ }^{2,11,16}$ Examples of multiparameter indices are the Surgical Pleth or Stress Index (which combines $\mathrm{HR}$ and $\mathrm{AP}$ ), ${ }^{9}$ the response index of nociception (which combines parameters from the electroencephalographic and hemodynamic signals), ${ }^{11}$ and the composite variability index (which combines the variability of the forehead electromyogram and BIS of the electroencephalogram). ${ }^{11}$ In the current study, we applied the multiparameter NoL to assess nociceptive responses at three levels of increasing nociceptive intensity. The NoL showed a remifentanil dose-independent increase in noxious response from -1.1 (nonnoxious stimulus) to 8.0 (incision) and 18.0 (intubation), with significant increases ( $\Delta$ signal) occurring for incision and intubation (fig. 1). This contrasts with the two other variables that were tested, the commonly used HR and MAP, which on a population level did show significant increases at intense stimulation but not at moderate noxious stimulation. The ROC curves (figs. 2 and 3; table 2) showed that of all tested $\Delta$ signals, $\Delta \mathrm{NoL}$ was best at differentiating between noxious and nonnoxious stimuli (AUC, 0.95). Similar observations were made for NoL (AUC, 0.82) relative to HR (0.66) and MAP (0.73). Cutoff values of 16 for NoL yielded an acceptable sensitivity of $73 \%$ with specificity of $80 \%$ and could be interpreted as a cutoff for discriminating noxious from nonnoxious stimuli. Furthermore, when testing the effect of multiple target concentrations of remifentanil, the NoL, in contrast to HR and MAP, remained unaffected under nonnociceptive conditions. This indicates that the NoL was a more reliable measure of nociception per

Table 3. AUC, Sensitivity, PPV, and NPV of the NoL, $\triangle \mathrm{NoL}, \mathrm{HR}, \triangle \mathrm{HR}, \mathrm{MAP}$, and $\triangle \mathrm{MAP}$ at a Specificity of $75 \%$

\begin{tabular}{|c|c|c|c|c|c|}
\hline Variable & AUC (95\% Cl) & Sensitivity (\%) & Specificity (\%) & PPV (\%) & NPV (\%) \\
\hline HR & $0.66(0.56-0.75)$ & 63 & 75 & 72 & 65 \\
\hline MAP & $0.73(0.64-0.81)$ & 63 & 75 & 75 & 64 \\
\hline NoL & $0.82(0.75-0.89)^{\star}$ & 73 & 75 & 75 & 72 \\
\hline$\Delta \mathrm{HR}$ & $0.84(0.77-0.91)$ & 84 & 75 & 78 & 81 \\
\hline$\triangle M A P$ & $0.78(0.70-0.86)$ & 75 & 75 & 78 & 72 \\
\hline$\Delta \mathrm{NoL}$ & $0.95(0.91-0.99) \dagger$ & 94 & 75 & 80 & 92 \\
\hline Random classifier & 0.50 & 25 & 75 & 50 & 50 \\
\hline
\end{tabular}

Statistical test was performed according to Hanley and McNeil. ${ }^{20} \mathrm{NoL}$, MAP, and HR given were obtained after noxious stimulation.

${ }^{*} P=0.001$ vs. HR; $P=0.036$ vs. MAP. $† P=0.0003$ vs. $\Delta \mathrm{HR} ; P<0.001$ vs. $\triangle \mathrm{MAP} ; P=0.0001$ vs. $\Delta \mathrm{NoL} ; P<0.0001$ vs. HR; $P<0.0001$ vs. MAP.

$\mathrm{AUC}=$ area under the receiver operating characteristic curve; HR = heart rate; $\mathrm{MAP}=$ mean arterial pressure; NoL $=$ nociception level; NPV = negative predictive value; PPV = positive predictive value. 
se, whereas HR and MAP are additionally affected by the hemodynamic effects of remifentanil (figs. 4-6).

Treister et al..$^{16}$ studied the same index response to three intensities of noxious stimulation in awake volunteers. Although none of the signals that make up the NoL were able to discriminate between the different noxious intensities, they observed, in agreement with our findings, that the combination of parameters (i.e., the NoL) was able to differentiate between pain and no pain and also between all three noxious intensities. This indicates that the NoL performs equally well in the awake and anesthetized individuals. Interestingly, single indices, such as HRV, perform better under conditions of general anesthesia than the awake state. ${ }^{24} \mathrm{Also}$ in chronic pain patients, the ability to obtain objective and accurate measures of pain and nociception, next to subjective self-reports (that are often colored by a variety of biopsychosocial factors), is important. ${ }^{21}$ To assess the ability of the NoL to track nociception in patients with chronic pain, Ben-Israel et al. ${ }^{25}$ studied patients with chronic radicular pain treated with spinal cord stimulation (SCS). The NoL values were in accordance with the efficacy of the SCS treatment as presented a correlation between reported pain score while turning the SCS device on and off. This indicates that the multivariate NoL may be used as an objective measurement of pain in patients with chronic pain and evaluate the efficacy of treatment.

The NoL is based on the advanced statistical and machine learning techniques to combine multiple signals into a single composite index. Machine learning methods rely on the concept that a specific algorithm that connects input to output can be trained to discover their optimal relationship. In our case, the link between input and output was established in a previous learn study, where the input were the records of physiologic signals collected during surgery under general anesthesia and the output the CISA, which is the linear combination of stimulus intensity and estimated analgesic plasma concentration. ${ }^{2}$ A detailed description of the CISA can be found in Ref. 2. The different autonomic variables that make up the NoL represent
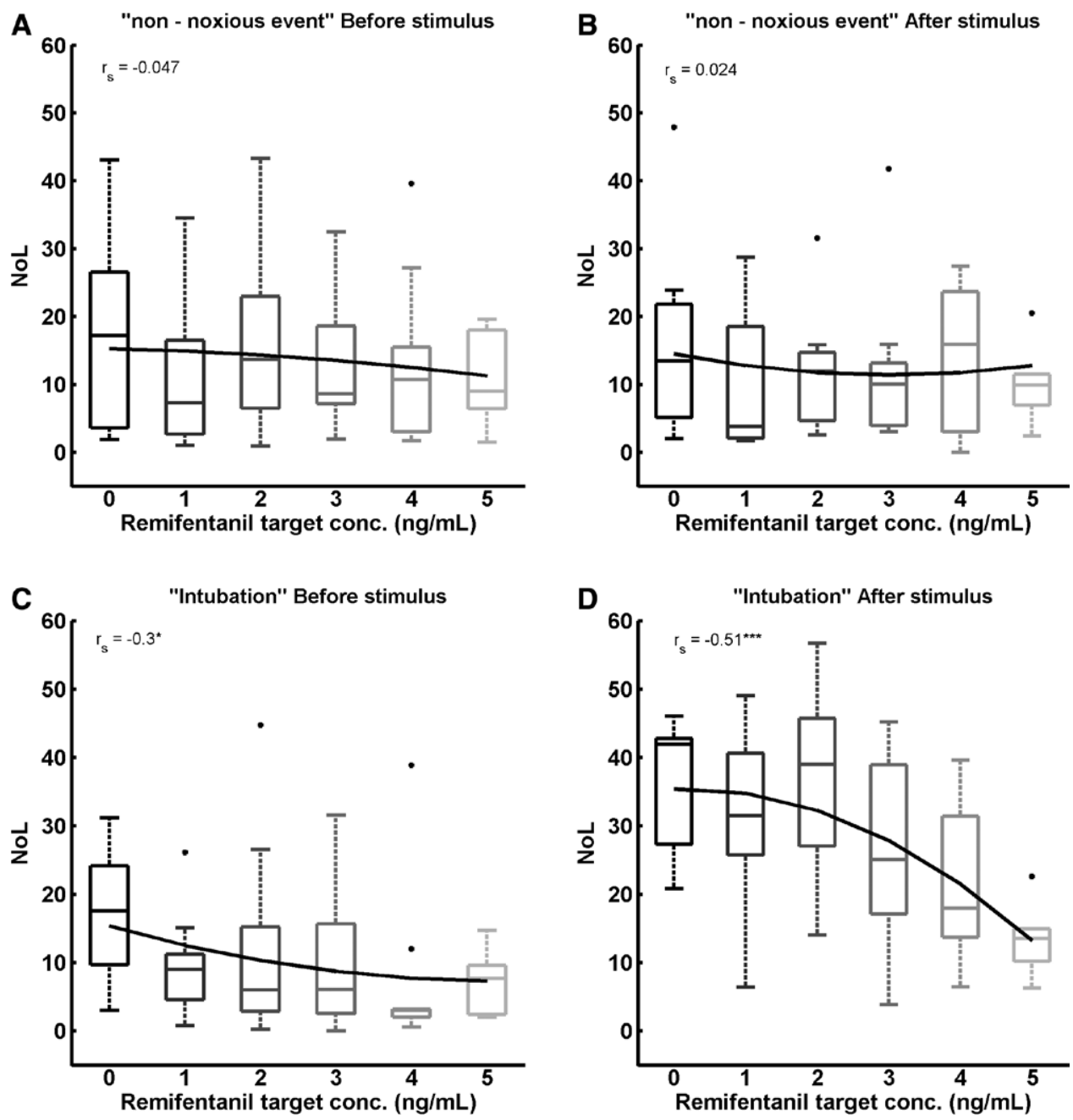

Fig. 4. Boxplot of the effect of remifentanil on nociception level (NoL) before $(A)$ and after $(B)$ noxious stimulation for nonnociceptive conditions and before $(C)$ and after $(D)$ noxious stimulation for intubation. Boxplots represent the median and 25 th and 75th percentiles, and the whiskers extend to the most extreme data points; outliers are plotted individually (block dots). The Spearman correlation is given $\left(r_{S}\right)$, with ${ }^{*} P<0.05$ and ${ }^{* * *} P<0.001$. A quadratic polynomial is fitted to the data to guide the eye. 

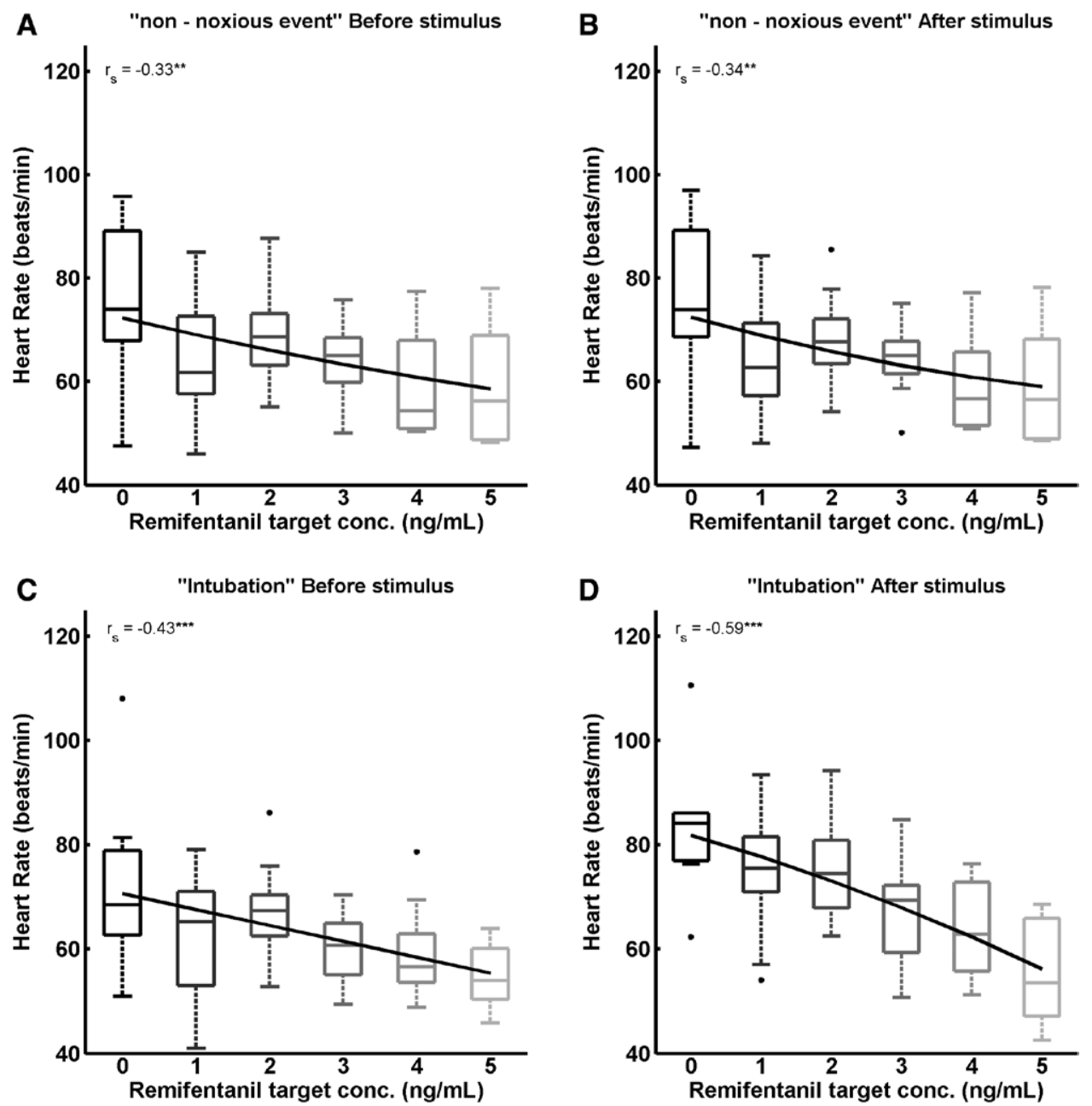

Fig. 5. Boxplot of the effect of remifentanil on heart rate before $(A)$ and after $(B)$ noxious stimulation for nonnociceptive conditions and before $(C)$ and after $(D)$ noxious stimulation for intubation. Boxplots represent the median and 25th and 75 th percentiles, and the whiskers extend to the most extreme data points; outliers are plotted individually (black dots). The Spearman correlation is given $\left(r_{\mathrm{S}}\right)$, with ${ }^{\star \star} P<0.01$ and ${ }^{* \star *} P<0.001$. A quadratic polynomial is fitted to the data to guide the eye.

different underlying systems, which have nontrivial nonlinear interactions. Adding their time derivatives to the equation (which is done to increase the ability to obtain a more reliable estimate of nociception) introduces an additional level of complexity by significantly enlarging the number of variables and creating additional nonlinear dependencies. Machine learning was performed using random forest regression analysis, a technique that is able to handle a large number of predictors to discover the optimal algorithm combining input to output, without the need for an a priori specification of a stochastic data model (i.e., the created algorithmic model treats the data mechanisms as unknown). ${ }^{12,13}$ Random forest regression is applied in different areas of science and engineering such as the identification of the smallest possible set of genes that can still achieve a good predictive outcome in clinical tests, prediction of protein interactions, and forecasting murderous conduct by individuals on probation or parole. ${ }^{26-28}$

An important issue is whether continuous measurement of nociception during surgery and treatment of signs of increased nociception will improve patient outcome. Chen et al. ${ }^{9}$ addressed this issue by studying the effect of surgical stress index-guided propofol-remifentanil anesthesia in patients undergoing elective ear, nose, and throat surgery. Compared with standard of practice, nociception-guided anesthesia reduced remifentanil consumption and unwanted movement and hemodynamic events. Parker et al. ${ }^{29}$ showed that the catecholamine (stress) response during anesthesia and emergence, in patients undergoing lower extremity revascularization, contributed to the development of postoperative hypertension and possibly also to the occurrence of thrombotic events. Similarly, also anesthesia with overdoses in anesthetic and opioid delivery may be associated with a poor outcome. ${ }^{30,31}$ Cumulatively these data suggest that the prevention of overdosing or underdosing of opioid and anesthetic drug delivery by continuous monitoring of nociception (and possibly also indices from the electroencephalogram) may result in a more stable nociceptive 

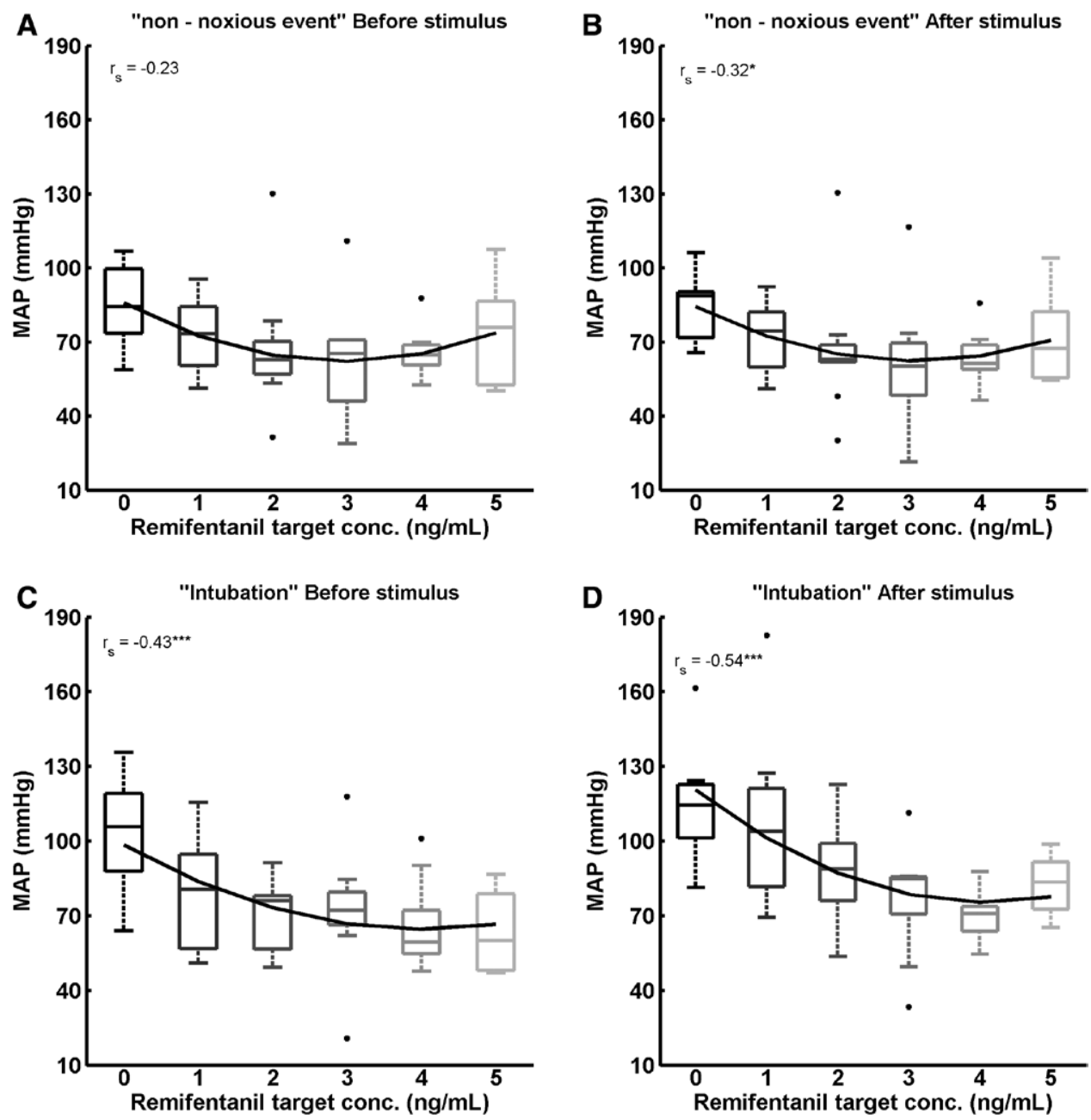

Fig. 6. Boxplot of the effect of remifentanil on mean arterial pressure (MAP) before $(A)$ and after $(B)$ noxious stimulation for nonnociceptive conditions and before $(C)$ and after $(D)$ noxious stimulation for intubation. Boxplots represent the median and 25th and 75th percentiles, and the whiskers extend to the most extreme data points; outliers are plotted individually (black dots). The Spearman correlation is given $\left(r_{s}\right)$ with ${ }^{*} P<0.05$ and ${ }^{* \star \star} P<0.001$. A quadratic polynomial is fitted to the data to guide the eye.

condition with beneficiary effects on outcome. Evidently, further studies are needed to fully understand the complexities of anesthetic monitoring and outcome.

The current study has some limitations: (1) the level of anesthetic depth as measured by the BIS was fixed to values ranging between 40 and 50. Therefore, we remain unaware of the influence of variations in anesthetic depth at multiple target opioid concentrations on the NoL. Assuming a synergistic effect of remifentanil and propofol on nociception, ${ }^{32}$ we expect some effect of propofol on the NoL. Our subprotocol was intended to study this issue but was not completed. Data were collected in four patients (two at BIS 30 and two at BIS 70). The analysis of these data suggests no dependency on BIS value on the effect of intubation on the NoL (data not shown). Evidently, this issue requires further study. (2) Each subject only received one dose level of remifentanil. This precluded the assessment of the intraindividual variance in the response to remifentanil. (3) We used a finger cuff system (Nexfin) to noninvasively measure the beat-to-beat blood pressure rather than invasive blood pressure. This finger cuff technique is reliable, ${ }^{17}$ and Nexfin MAP measurements were second to the NoL in ability to detect nociceptive responses (fig. 2). Possibly adding the beat-to-beat MAP to the NoL algorithm would further improve the accuracy of the NoL. (4) We performed our studies in 72 patients. Because of technical, logistic, or other issues, nonnoxious, incision, and intubation events were obtained on average in 60 $(83 \%)$ subjects. This may have affected the outcome of the study. Given the fact that the NoL performed as expected from results of previous studies, the loss of data did not affect the power of our study with respect to the NoL. ${ }^{2,16}$ Conversely, the loss of data may have caused the inability of MAP to detect moderate nociceptive stimuli (fig. 1). (5) Finally, we excluded patients on $\beta$-adrenergic-blocking drugs. Because $\beta$-adrenergic blockers and other vasoactive drugs affect the autonomic system at multiple sites, their effect on the accuracy of the NoL requires further study. 
In conclusion, we applied a novel multidimensional index, the NoL, to detect nociception during conditions of no, moderate, and intense noxious stimulation in surgical patients under propofol-remifentanil anesthesia. We observed that compared with HR and MAP, the index was best at differentiating nociceptive from nonnociceptive conditions. In additionally, in contrast to MAP and HR, the NoL remained unaffected by the hemodynamic effects of increasing concentrations of remifentanil.

\section{Acknowledgments}

The authors thank Sefik Karagozoglu, M.D., Jetske van der Bos, M.D., and Anna Westgaard, M.D., Anesthesia Residents at Leiden University Medical Center, Leiden, The Netherlands, for their assistance and help with recruitment of patients and performance of the experiments.

This investigator-initiated trial was supported by institutional and departmental funds. Medasense Biometrics Ltd. (Ramat Yishai, Israel) provided the hardware and software used in the study.

\section{Competing Interests}

Dr. Dahan received consultancy fee from Medasense Biometrics Ltd. (Ramat Yishai, Israel). The other authors declare no competing interests.

\section{Correspondence}

Address correspondence to Dr. Dahan: Department of Anesthesiology, Leiden University Medical Center, P5-Q, 2300 RC Leiden, The Netherlands. a.dahan@lumc.nl. This article may be accessed for personal use at no charge through the Journal Web site, www.anesthesiology.org.

\section{References}

1. Loeser JD, Treede RD: The Kyoto protocol of IASP Basic Pain Terminology. Pain 2008; 137:473-7

2. Ben-Israel N, Kliger M, Zuckerman G, Katz Y, Edry R: Monitoring the nociception level: A multi-parameter approach. J Clin Monit Comput 2013; 27:659-68

3. Boselli E, Daniela-Ionescu M, Bégou G, Bouvet L, Dabouz R, Magnin C, Allaouchiche B: Prospective observational study of the non-invasive assessment of immediate postoperative pain using the analgesia/nociception index (ANI). Br J Anaesth 2013; 111:453-9

4. Storm H: Changes in skin conductance as a tool to monitor nociceptive stimulation and pain. Curr Opin Anaesthesiol 2008; 21:796-804

5. Shimoda O, Ikuta Y, Skamoto M, Terasaki H: Skin vasomotor vasomotor reflex predict circulatory responses to laryngoscopy and intubation. Anesthesiology 1998; 88:297-304

6. Vanluchene AL, Struys MM, Heyse BE, Mortier EP: Spectral entropy measurement of patient responsiveness during propofol and remifentanil. A comparison with the bispectral index. Br J Anaesth 2004; 93:645-54

7. Larson MD, Kurz A, Sessler DI, Dechert M, Bjorksten AR, Tayefeh F: Alfentanil blocks reflex pupillary dilation in response to noxious stimulation but does not diminish the light reflex. AnEsthesiology 1997; 87:849-55

8. Bonhomme V, Uutela K, Hans G, Maquoi I, Born JD, Brichant JF, Lamy M, Hans P: Comparison of the surgical Pleth Index ${ }^{\mathrm{TM}}$ with haemodynamic variables to assess nociception-anti-nociception balance during general anaesthesia. Br J Anaesth 2011; 106:101-11

9. Chen X, Thee C, Gruenewald M, Wnent J, Illies C, Hoecker J, Hanss R, Steinfath M, Bein B: Comparison of surgical stress index-guided analgesia with standard clinical practice during routine general anesthesia: A pilot study. AnEsthesiology 2010; 112:1175-83

10. Sahinovic MM, Eleveld DJ, Kalmar AF, Heeremans EH, De Smet T, Seshagiri CV, Absalom AR, Vereecke HE, Struys MM: Accuracy of the composite variability index as a measure of the balance between nociception and antinociception during anesthesia. Anesth Analg 2014; 119:288-301

11. Rantanen M, Yli-Hankala A, van Gils M, Yppärilä-Wolters $H$, Takala P, Huiku M, Kymäläinen M, Seitsonen E, Korhonen I: Novel multiparameter approach for measurement of nociception at skin incision during general anaesthesia. $\mathrm{Br} \mathrm{J}$ Anaesth 2006; 96:367-76

12. Breiman L: Statistical modelling: The two cultures. Stat Sci 2001; 16:199-231

13. Breiman L: Random forests. Mach Learn 2001; 45:5-32

14. Minto CF, Schnider TW, Egan TD, Youngs E, Lemmens HJ, Gambus PL, Billard V, Hoke JF, Moore KH, Hermann DJ, Muir KT, Mandema JW, Shafer SL: Influence of age and gender on the pharmacokinetics and pharmacodynamics of remifentanil. I. Model development. AnEsThesiology 1997; 86:10-23

15. Marsh B, White M, Morton N, Kenny GN: Pharmacokinetic model driven infusion of propofol in children. Br J Anaesth $1991 ; 67: 41-8$

16. Treister R, Kliger M, Zuckerman G, Goor Aryeh I, Eisenberg E: Differentiating between heat pain intensities: The combined effect of multiple autonomic parameters. Pain 2012; 153:1807-14

17. Martina JR, Westerhof BE, van Goudoever J, de Beaumont EM, Truijen J, Kim YS, Immink RV, Jöbsis DA, Hollmann MW, Lahpor JR, de Mol BA, van Lieshout JJ: Noninvasive continuous arterial blood pressure monitoring with Nexfin ${ }^{\circ}$. Anesthesiology 2012; 116:1092-103

18. Ausems ME, Hug CC Jr, Stanski DR, Burm AG: Plasma concentrations of alfentanil required to supplement nitrous oxide anesthesia for general surgery. ANESTHESIOLOGY 1986; 65:362-73

19. Gelb AW, Leslie K, Stanski DR, Shafer SL: Monitoring the depth of anesthesia, Miller's Anesthesia, 7th edition. Edited by Miller RD, Eriksson LI, Fleisher LA, Wiener-Kronish JP, Young WL. Philadelphia, Churchill Livingstone, 2009, pp 1229-66

20. Hanley JA, McNeil BJ: A method of comparing the areas under receiver operating characteristic curves derived from the same cases. Radiology 1983; 148:839-43

21. Loggia ML, Napadow V: Multi-parameter autonomic-based pain assessment: More is more? Pain 2012; 153:1779-80

22. Jeanne M, Logier R, De Jonckheere J, Tavernier B: Heart rate variability during total intravenous anesthesia: Effects of nociception and analgesia. Auton Neurosci 2009; 147:91-6

23. Loggia ML, Juneau M, Bushnell MC: Autonomic responses to heat pain: Heart rate, skin conductance, and their relation to verbal ratings and stimulus intensity. Pain 2011; 152:592-8

24. Jeanne M, Delecroix M, De Jonckheere J, Keribedj A, Logier $\mathrm{R}$, Tavernier B: Variations of the analgesia nociception index during propofol anesthesia for total knee replacement. Clin J Pain 2014; 30:1084-8

25. Ben-Israel N, Amos Y, Kliger M, Racheli N, Zuckerman G, Tresiter R, Suzan E, Eisenberg E: Objective assessment of spinal cord stimulation effectiveness on chronic radicular pain. Poster presented at: 15th World Congress of Pain, Buenos Aires, Argentina, October 10, 2014. Available at: http://www.abstractsonline. com/Plan/ViewAbstract.aspx?sKey=31e 783c1-a5ec-4f59bb50-493f3ac275bb\&cKey=28618cef-2457-435d-be2a- 
$\mathrm{d} 0114 \mathrm{f} 1 \mathrm{fb} 907 \& \mathrm{mKey}=83 \mathrm{f} 2864 \mathrm{e}-03 \mathrm{e} 9-4 \mathrm{e} 87-8194-727 \mathrm{~d} 1 \mathrm{~d} 8 \mathrm{f} 65 \mathrm{e} 0$. Accessed April 15, 2015

26. Díaz-Uriarte R, Alvarez de Andrés S: Gene selection and classification of microarray data using random forest. BMC Bioinformatics 2006; 7:3

27. Chen XW, Liu M: Prediction of protein-protein interactions using random decision forest framework. Bioinformatics 2005; 21:4394-400

28. Berk R, Sherman L, Barnes G, Kurtz E, Ahlman L: Forecasting murder within a population of probationers and parolees: A high stakes application of statistical learning. J R Statist Soc 2009; 172:1-21

29. Parker SD, Breslow MJ, Frank SM, Rosenfeld BA, Norris EJ, Christopherson R, Rock P, Gottlieb SO, Raff H, Perler B, Williams GM: Catecholamine and cortisol response to lower extremity revascularization: Correlation with outcome variables. Crit Care Med 1995; 23:1954-61

30. Monk TG, Saini V, Weldon BC, Sigl JC: Anesthetic management and one-year mortality after noncardiac surgery. Anesth Analg 2005; 100:4-10

31. Sessler DI, Sigl JC, Kelley SD, Chamoun NG, Manberg PJ, Saager L, Kurz A, Greenwald S: Hospital stay and mortality are increased in patients having a "triple low" of low blood pressure, low bispectral index, and low minimum alveolar concentration of volatile anesthesia. ANESTHESIOLOGY 2012; 116:1195-203

32. Dahan A, Niesters M, Smith T, Overdyk F: Opioids, Clinical Anesthesia. Edited by Barash PG, Cullen BF, Stoelting RK, Cahalan MK, Stock MC, Ortega R. Philadelphia, Wolters Kluwer, Lippincot Williams \& Wilkins. 2013, pp 501-22 